Prons

trobertivier Journal of Nonlinear Mathematical Physics

\title{
Mean-Field Limit of a Microscopic Individual-Based Model Describing Collective Motions
}

Carlo Bianca, Christian Dogbe

To cite this article: Carlo Bianca, Christian Dogbe (2015) Mean-Field Limit of a Microscopic Individual-Based Model Describing Collective Motions, Journal of Nonlinear Mathematical Physics 22:1, 117-143, DOI:

https://doi.org/10.1080/14029251.2015.996444

To link to this article: https://doi.org/10.1080/14029251.2015.996444

Published online: 04 January 2021 


\title{
Mean-Field Limit of a Microscopic Individual-Based Model Describing Collective Motions
}

\author{
Carlo Bianca \\ Laboratoire de Physique Théorique de la Matière Condensée, CNRS and Sorbonne Universités, \\ UPMC Univ Paris 06, UMR 7600 4, place Jussieu, case courrier 121, 75252 Paris cedex 05, France \\ bianca@lptmc.jussieu.fr \\ Christian Dogbe \\ Département de Mathématiques, Université de Caen Basse-Normandie, \\ Laboratoire de Mathématiques Nicolas Oresme, LMNO \\ CNRS, UMR 6139, 14032 Caen Cedex, France \\ christian.dogbe@unicaen.fr
}

Received 10 May 2014

Accepted 6 October 2014

\begin{abstract}
This paper is mainly concerned with a mean-field limit and long time behavior of stochastic microscopic interacting particles systems. Specifically we prove that a class of ODE modeling collective interactions in animals or pedestrians converges in the mean-field limit to the solution of a non-local kinetic PDE. The mathematical analysis, performed by weak measure solutions arguments, shows the existence of measure-valued solutions, asymptotic stability and chaos propagation that are relevant properties in the description of collective behaviors that emerge in animals and pedestrians motions.
\end{abstract}

Keywords: Collective motion; interacting stochastic particle systems; weak solutions; uniqueness.

2000 Mathematics Subject Classification: 82C22，35Q35，60K35，35Q83 35A05

\section{Introduction}

The emergence of collective behavior in large particle systems has recently attracted much attention considering that this behavior arises in many physical systems of the real-world such as in crowd and swarm dynamics, social and economic systems. In this context, mathematical models at the microscopic, mesoscopic and macroscopic scales, have been proposed in an attempt to obtain an analytical and multiscale description of the phenomena.

This paper deals with a qualitative analysis of a class of models for pedestrian dynamics and specifically with mathematical models that can be approximated by mean-field particle interactions. The main question addresses is whether, for a given microscopic individual-based model, the corresponding macroscopic (mean-field) dynamics can be derived.

The mathematical study of such problems goes back to the last century. In particular, in the context of classical mechanics where the mean-field limit is described by the Vlasov or Vlasov-type equation, the problem has been rigorously analyzed by Braun and Hepp in [7] and by Neunzert in [25]. The interested reader is referred to the recent lecture notes by Golse [18] for a more deeper understanding of the subject, see also [17]. In particular the mean-field limit proposed in the present paper is performed according to papers $[17,18]$. 
The underlying microscopic model is a system of $N$-indistinguishable individuals (pedestrians, birds, fish) whose microscopic state includes position and velocity variables. For each position variable $x_{i}$ we introduce the velocity variable $v_{i}=\dot{x}_{i}$ (time-derivative of the position), so that the whole state of the system at time $t$ is described by $\left(x_{1}, v_{1}\right), \ldots,\left(x_{N}, v_{N}\right)$. The domain of positions is $\mathbb{R}^{d}$ or a subset of $\mathbb{R}^{d}$ and the domain of velocities is $\mathbb{R}^{d}(d=2$ or $d=3$ for crowd or swarm dynamics). However, the approach proposed in this paper is quite general to allow the modeling of various systems composed by interacting agents even in higher dimensions. Thus in the whole paper the dimension $d$ will be let arbitrary. Accordingly the microscopic state of the $i$ th particle at time $t$ is denoted by $z_{i}=\left(x_{i}, v_{i}\right) \in \mathbb{R}^{2 d}$. The interaction between the $i$ th and the $j$ th particle is modeled by the function $K: \mathbb{R}^{d} \times \mathbb{R}^{d} \rightarrow \mathbb{R}^{d}$ The particle dynamics is describeb by the following system of $d \times N$ ODEs (see $[9,22,27])$ :

$$
\left\{\begin{array}{l}
\dot{z}_{i}(t)=\mathbb{G}\left(z_{i}\right)+\frac{1}{N} \sum_{j=1}^{N} K\left(z_{i}(t), z_{j}(t)\right), \quad i=1, \ldots, N \\
z_{i}(0)=z_{j}^{i n}
\end{array}\right.
$$

where the term $\mathbb{G}\left(z_{i}\right)$ models the interactions inside the system, i.e. the external forces acting on the $i$ th particle that do not depend on the other particles. The coefficient $1 / N$ is the so-called "weak coupling". This interaction among the particles is "weak", which means that as the particle number $N$ goes to infinity, the range of the interaction remains fixed, whereas its strength is rescaled by $1 / N$. Having in mind this limiting behaviour, it seems convenient to describes a particle in $x \in \mathbb{R}^{d}$ by $(1 / N) \delta_{x}$ (i.e. the Dirac measure with mass $1 / N$ concentrated at $x$ ). A preliminary difficulty in dealing with the mean-field limit of (1.1) is the singularity of $K(z)$ for $z$ close to 0 .

The main aim that wants to be pursued in this paper refers to the case $N \rightarrow \infty$. Specifically, in what timescale this limit exist?

In the large limit, $N \rightarrow \infty$, of (1.1) it is expected that

$$
\frac{1}{N} \sum_{j=1}^{N} K\left(z_{i}(t), z_{j}(t)\right) \rightarrow \int_{\mathbb{R}^{d}} K\left(z_{i}(t), z_{j}(t)\right) f(t, d z)
$$

and that the $N$-particle system of differential equations (1.1) can be replaced with the following single differential equation

$$
\begin{aligned}
\dot{z}(t) & =\mathbb{G}(z)+\int_{\mathbb{R}^{d}} K\left(z(t), z^{\prime}(t)\right) f(t, d z) \\
& :=\mathbb{G}(z)+\mathbf{F}[f](t, x),
\end{aligned}
$$

where $f(t, d z)$ is unknown as is $z(t)$; so that it seems that this single differential equation is insufficient to determine both these unknowns.

Bearing all above in mind, the underlying macroscopic framework is the following conservation law:

$$
\left\{\begin{array}{l}
\partial_{t} f(t, z)+\operatorname{div}_{z}(\mathscr{K}[f](t, z) f(t, z))=0, \quad z \in \mathbb{R}^{d}, t \in \mathbb{R} \\
f(0, z)=f_{0}(z),
\end{array}\right.
$$


where $f(t, z)$ is a probability density, $z \in \mathbb{R}^{d}, t$ is the time variable and $\mathscr{K}[f] \equiv \mathscr{K}[f](t, z) \in \mathbb{R}^{d}$ is the following non-linear functional operator:

$$
\mathscr{K}[f](t, z)=\mathbb{G}(z)+\int_{\mathbb{R}^{d}} K\left(z, z^{\prime}\right) f\left(d z^{\prime}\right),
$$

where $K$ is a given, smooth, vector-valued kernel, whose explicit form depends on the system under consideration. Here, div $\mathscr{K}(t, \cdot)$ is understood in distributional sense.

The contents of the present paper is divided into six more sections which follow this introduction. In detail, in Section 2 we set the notations and some backgrounds about the mathematical problem. Section 3 is concerned with the definition and the existence of measure-valued solutions to (1.4). Section 4 provides results on the mean field characteristic flow. Section 5 deals with some stability results. Section 6 provides the stochastic formulation of the problem where we prove the propagation of chaos result related to our problem. In this context, the word "chaotic" is used as a synonym for "independent" and identically distributed. Finally, Section 7 highlights conclusions and applications.

\section{Notation and Background}

This section is devoted to the additional notation and known results that will be used in the next sections. Specifically the following notations for functional spaces will be considered:

- For each topological space $X$ and each finite dimensional vector space $E$ on $\mathbb{R}$, we denote by $C(X, E)$ the set of continuous functions defined on $X$ with values in $E$, and by $C_{c}(X, E)$ the set of functions belonging to $C(X, E)$ whose support is compact in $X$. For each $d, k \geq 1$, we denote by $C_{c}^{k}\left(\mathbb{R}^{d}, E\right)$ the set of functions of class $C^{k}$ defined on $\mathbb{R}^{d}$ with values in $E$ whose support is compact in $\mathbb{R}^{d}$. We also define by $C(X):=C(X, \mathbb{R}), C_{c}(X):=C_{c}(X, \mathbb{R})$ and $C_{c}^{k}\left(\mathbb{R}^{d}\right):=C_{c}^{k}\left(\mathbb{R}^{d}, \mathbb{R}\right)$.

- For each topological space $X$ and each finite dimensional vector space $E$ on $\mathbb{R}$, we denote by $C_{b}(X, E)$ the set of continuous functions defined on $X$ with values in $E$ that are bounded on $X$. For each $d, k \geq 1$, we denote by $C_{b}^{k}\left(\mathbb{R}^{d}, E\right)$ the set of functions of class $C^{k}$ defined on $\mathbb{R}^{d}$ with values in $E$, whose partial derivatives are bounded on $\mathbb{R}^{n}$ : for each norm $|\cdot|_{E}$ on $E$, one has

$$
C_{b}^{k}\left(\mathbb{R}^{d}, E\right):=\left\{f \in C^{k}\left(\mathbb{R}^{d}, E\right) \text { s.t. } \sup _{x \in \mathbb{R}^{d}}\left|\partial^{\alpha} f(x)\right|_{E}<\infty \text { for each } \alpha \in \mathbb{N}^{n}\right\}
$$

- We denote by $\mathscr{P}_{r}\left(\mathbb{R}^{d}\right)$, for each $r>0$, the set of Borel probability measures $\mu$ on $\mathbb{R}^{d}$ such that

$$
\int_{\mathbb{R}^{d}}|z|^{r} \mu(d z)<\infty
$$

Henceforth, the set of Borel probability measures on $\mathbb{R}^{d}$ will be denoted by $\mathscr{P}\left(\mathbb{R}^{d}\right)$.

Monge-Kantorovich-Wasserstein distance. First, we model transference plans by probability measures $\pi$ on the product space $\mathbb{R}^{d} \times \mathbb{R}^{d}$. Given $\mu, v \in \mathscr{P}_{p}\left(\mathbb{R}^{d}\right)$ defined respectively on some measure 
spaces $\mathbb{R}^{d}$, we define

$$
\Pi_{\mu, v}=\Pi(\mu, v):=\left\{\pi \in \mathscr{P}\left(\mathbb{R}^{d} \times \mathbb{R}^{d}\right) ; \int_{\mathbb{R}^{d}} d \pi(x, y)=d \mu(x), \int_{\mathbb{R}^{d}} d \pi(x, y)=d v(y)\right\} .
$$

$\Pi(\mu, v)$ is the set of Borel probability measures $\pi$ on $X \times Y$ with first and second marginals $\mu$ and $v$ respectively. Equivalently, for each $\pi \in \mathscr{P}\left(\mathbb{R}^{d} \times \mathbb{R}^{d}\right)$,

$$
\pi \in \Pi(\mu, v) \Leftrightarrow \iint_{\mathbb{R}^{d} \times \mathbb{R}^{d}}(\phi(x)+\psi(y)) \pi(d x d y)=\int_{\mathbb{R}^{d}} \phi(x) \mu(d x)+\int_{\mathbb{R}^{d}} \psi(y) v(d y)
$$

for each $\phi, \psi \in C\left(\mathbb{R}^{d}\right)$ such that $\phi(z)=O\left(|z|^{p}\right)$ and $\psi(z)=O\left(|z|^{p}\right)$ as $|z| \rightarrow \infty$.

For each $p \geqslant 1$ and each $\mu, v \in \mathscr{P}_{r}\left(\mathbb{R}^{d}\right)$, the Monge-Kantorovich-Wasserstein distance $W_{p}(\mu, v)$ between $\mu$ and $v$ is defined by the formula

$$
W_{p}(\mu, v)=\inf \left\{\mathbb{E}\left[|X-Y|^{p}\right]^{1 / p}, \mathscr{L} \text { aw }(X)=\mu, \quad \mathscr{L} \text { aw }(Y)=v\right\},
$$

where the infimum is taken on all the couples $(X, Y)$ of random variables with values in $\mathbb{R}^{d}$ and $|\cdot|$ is distance on $\mathbb{R}^{d}$. In the integral form (2.2) reads:

$$
W_{p}(\mu, v)=\inf _{\pi \in \Pi(\mu, v)}\left(\iint_{\mathbb{R}^{d} \times \mathbb{R}^{d}}|x-y|^{p} \pi(d x d y)\right)^{1 / p} .
$$

The following Proposition holds.

Proposition 2.1. The Monge-Kantorovich-Wasserstein distance with exponent 1 is also given by the the following formula

$$
W_{1}(\mu, v)=\sup _{\substack{\phi \in \operatorname{Lip}\left(\mathbb{R}^{d}\right) \\ \operatorname{Lip}(\phi) \leq 1}}\left|\int_{\mathbb{R}^{d}} \phi(z) \mu(d z)-\int_{\mathbb{R}^{d}} \phi(z) v(d z)\right|,
$$

where

$$
\operatorname{Lip}(\phi):=\sup _{\substack{x \neq y \\ x, y \in \mathbb{R}^{d}}} \frac{|\phi(x)-\phi(y)|}{|x-y|}
$$

is the Lipschitz constant of $\phi$.

The reader will note that in general, in the literature, one finds the definition of the distance from Wasserstein with $p=2$. It is a distance inducing weak convergence. The less hilbertian distance is when $p=1$ and it is perhaps also more natural, since it is that which consists in saying that it is the trace on the space of the probabilities, on the standard $W^{1, \infty}$ (Theorem of Kantorovitch-Silverstein). The map $d \mapsto d_{M K}$ is a metric that induces weak convergence and increasing with respect $p$ (that is Jensen's inequality).

The notation $w-\mathscr{M}\left(\mathbb{R}^{d}\right)$ denotes the set of Radon measures on $\mathbb{R}^{d}$ equipped with its weak topology. It is well known (see for example [14]) that $d$ metrizes the weak convergence in $\mathscr{M}\left(\mathbb{R}^{d}\right)$ (that is, the convergence in the duality with bounded continuous functions).

Notice that not all the material here comes from [23] (see also [31]). 
Push-forward measure. Given two measurable spaces $(X, \mathscr{X})$ and $(Y, \mathscr{Y})$, and let $T: X \rightarrow Y$ be an $(\mathscr{X}, \mathscr{Y})$-measurable map. One can then defines a positive measure on $(Y, \mathscr{Y})$, denoted $v=: T \# \mu$, and referred to as the push-forward (or image measure) of the measure $\mu$ under the map $T$, by requiring that the measurable sets on $\mathscr{Y}$ are the sets $B \subset \mathscr{Y}$ such that $T^{-1}(B)$ is measurable, and defining naturally

$$
v(B):=\mu\left(T^{-1}(B)\right)
$$

The rule that expresses the image measure in terms of integrals of continuous functions is as follows:

$$
\int_{Y} \mathbf{1}_{B}(y) v(d y)=\int_{X} \mathbf{1}_{T^{-1}(B)}(x) \mu(d x)=\int_{X} \mathbf{1}_{B}(T(x)) \mu(d x)
$$

recalling that

$$
\mathbf{1}_{T^{-1}(B)}(x)=\mathbf{1}_{B} \circ T .
$$

In the sequel, we will used the dual notation

$$
\langle\varphi, \mu\rangle=\int_{\mathbb{R}^{d} \times \mathbb{R}^{d}} \varphi d \mu .
$$

\subsection{Example of models}

Following the models of particle physics, we assume that the interactions among individuals are governed by an interacting potential. The spirit of our results is captured by the following examples.

- In the framework of crowd dynamics, the term $\mathbf{F}[f]$ models the effect of interactions with other pedestrians on the current velocity (see, for instance, $[4,5,16]$ ). We assume that $\mathbf{F}[f]$ has the form:

$$
\mathbf{F}[f](t, x):=\int_{\mathbb{R}^{d}-\{0\}} g\left(\frac{x-y}{|x-y|} \cdot \frac{v_{d}}{\left|v_{d}\right|}\right) f(|y-x|) \frac{y-x}{|y-x|} \rho(t, x) d \lambda^{d}(y),
$$

on the time-dependent mass measures $\lambda^{d}$, where $\rho$ represents the macroscopic density of $f$ :

$$
\rho(t, x):=\int f(t, x, v) d v
$$

In (2.3) we have used the following modeling ansätze:

- $g$ is a function from $[-\pi, \pi] \rightarrow[0,1]$ that encodes the fact that an individual perception is not equal in all directions.

- $f$ is a function that describes the effect of the mutual distance among individuals on their interaction.

Furthermore, we assume that the interaction is translation invariant, thus the force derives from an interaction potential. Omitting time dependence in the variables, $W: \mathbb{R}^{d} \rightarrow \mathbb{R}$ is the potential governing the interactions and $\nabla W$ corresponds to a (generalized) force exerted at position $x$ by a 
particle located at position $y$ and $\nabla W$ is equal to

$$
\nabla W(|x-y|):=W^{\prime}(|x-y|) \frac{x-y}{|x-y|} .
$$

To be more precise, we assume that there exists a function $F: \mathbb{R}^{+} \rightarrow \mathbb{R}$ such that $f=-F^{\prime}$. Then:

$$
\begin{aligned}
f(|y-x|) \frac{y-x}{|y-x|} & =-f(|x-y|) \frac{x-y}{|x-y|} \\
& =F^{\prime}(|x-y|) \frac{x-y}{|x-y|}=\nabla F(|x-y|)
\end{aligned}
$$

where the relation $\nabla|x-y|=\frac{x-y}{|x-y|}$ has been considered. Thus we have

$$
W(x-y):=F(|x-y|) .
$$

Finally, we write $\mathbf{F}[f]$ into the following form:

$$
\mathbf{F}\left[\rho_{f}\right](t, x)=(\nabla W \star \rho)(t, x)=\int_{\mathbb{R}^{d}} \nabla W(x-y) \rho_{t}(t, y) d y, \quad \forall t \in(0, T), \quad x \in \mathbb{R}^{d} .
$$

For the term $\mathbb{G}$, it is, for example, possible to have $v_{d e s}=a \in \mathbb{R}^{d}$; that is, the desired velocity has constant magnitude and direction. To comply with the assumption that $v_{d e s}$ can be written as $\nabla V$, we have to take $V(x)=a \cdot x$.

Therefore, we can assume that the field $\mathscr{K}[f]$ has the following form

$$
\mathscr{K}[f]:=\nabla V+\nabla W \star \rho
$$

where $V: \mathbb{R}^{d} \rightarrow \mathbb{R}$ is a confinement potential and $W: \mathbb{R}^{d} \rightarrow \mathbb{R}$ is a interaction potential. We can assume that $W$ is a symmetric function, that is

$$
W(-z)=W(z) \text { for all } \quad z \in \mathbb{R}^{d}
$$

- Another collective dynamics model is that obtained dividing the interaction term in (1.1) into two parts: the repulsion, still modeled as a gradient of the repulsion potential, and the attraction which, however, is not a potential gradient any more [21]:

$$
\mathscr{K}[f](t, x, v)=\left(a-b\left|v_{i}\right|^{2}\right) v_{i}-\frac{1}{N} \sum_{j \neq i} \nabla U_{R}\left(\left|x_{i}-x_{j}\right|\right)-\frac{1}{N} \sum_{j \neq i} f_{A}\left(\alpha\left(x_{i}, x_{j}\right)\right) \frac{x_{i}-x_{j}}{\left|x_{i}-x_{j}\right|} .
$$

\section{Measure Valued Solution}

The main idea is to replace the ODE system (1.1) by a PDE such that the computational cost of its solution does not grow as $N$ goes to infinity. The answer is given by the mean field approach. Let $f=f(t, x, v)$ be the one-particle distribution of the particles positioned at $(t, x) \in \mathbb{R}^{+} \times \mathbb{R}^{d}$ with a velocity $v \in \mathbb{R}^{d}$. The corresponding kinetic equation of (1.1), in the mean-field limit as $N \rightarrow \infty$, is 
the following Vlasov-type kinetic equation:

$$
\left\{\begin{array}{l}
\partial_{t} f(t, z)+\operatorname{div}_{z}(f(t, z) \mathscr{K} f(t, z))=0, \quad z \in \mathbb{R}^{d}, t \in \mathbb{R}, \\
f(0, z)=f_{0},
\end{array}\right.
$$

where $f_{0} \in L^{1}\left(\mathbb{R}^{d} ;(1+|z|) d z\right)$ and $\mathscr{K} f(t, z)$ denotes $(\mathscr{K} f(t, \cdot))(z)$, and

$$
\mathscr{K} \phi(z):=\mathbb{G}(z)+\int_{\mathbb{R}^{d}} K\left(z, z^{\prime}\right) \phi\left(z^{\prime}\right) d z^{\prime}, \quad \phi \in L^{1}\left(\mathbb{R}^{d} ;(1+|z|) d z\right) .
$$

The equation (3.1) is obtained through the mean-field limit, that is, when each particle feels the combined effect of all other particles.

Assume that

$$
\iint_{\mathbb{R}^{d} \times \mathbb{R}^{d}} f(0, x, v) d x d v=1 \quad \text { and } \iint_{\mathbb{R}^{d} \times \mathbb{R}^{d}} v f(0, x, v) d x d v=0 .
$$

Since the dynamics of the system (3.1) preserves the total mass and momentum, one has

$$
\iint_{\mathbb{R}^{3} \times \mathbb{R}^{3}} f(t, x, v) d x d v=1 \quad \text { and } \iint_{\mathbb{R}^{3} \times \mathbb{R}^{3}} v f(t, x, v) d x d v=1, \quad \forall t \geqslant 0 .
$$

Thus, the system (3.1) can be rewritten in the following form:

$$
\partial_{t} f+\operatorname{div}_{z}\left(f(t, z) \int_{\mathbb{R}^{2 d}} K\left(z, z^{\prime}\right) f\left(t, z^{\prime}\right) d z^{\prime}\right)=0
$$

with $z=(x, v)$ and

$$
K\left(z, z^{\prime}\right)=K\left((x, v),\left(x^{\prime}, v^{\prime}\right)\right):=\left(v-v^{\prime}, \mathbb{G}(x)+\nabla W \star \rho\right) .
$$

The existence and uniqueness of the solution of (3.3) will be obtained as a consequence of the construction of the mean-field flow.

Before formulating the main results, we will make the following assumptions on the interaction kernel $K$ (according to $[17,18]$ ):

(H1) $K: \mathbb{R}^{d} \times \mathbb{R}^{d} \rightarrow \mathbb{R}^{d}$ is a continuous map such that

$$
K\left(z, z^{\prime}\right)-K\left(z^{\prime}, z\right)=0, \quad z, z^{\prime} \in \mathbb{R}^{d},
$$

namely $K$ is symmetric. The assumption (3.5) is more restrictive of what is really necessary, but it has been set for the sake of simplicity.

Possibly, we can replace (3.5) by the following assumptions:

(H2) $K: \mathbb{R}^{d} \times \mathbb{R}^{d} \rightarrow \mathbb{R}^{d}$ is a continuous map satisfying

$$
K\left(z, z^{\prime}\right)+K\left(z^{\prime}, z\right)=0, \quad z, z^{\prime} \in \mathbb{R}^{d}
$$

(H3) $K \in C^{1}\left(\mathbb{R}^{d} \times \mathbb{R}^{d} ; \mathbb{R}^{d}\right)$, with bounded partial derivatives of order 1 . In other words, there exists a constant $L \geqslant 0$ such that

$$
\sup _{z^{\prime} \in \mathbb{R}^{d}}\left|\nabla_{z} K\left(z, z^{\prime}\right)\right| \leqslant L, \quad \text { and } \quad \sup _{z \in \mathbb{R}^{d}}\left|\nabla_{z^{\prime}} K\left(z, z^{\prime}\right)\right| \leqslant L .
$$


(H4) We assume that $\mathbb{G}$ is in $\operatorname{Lip}\left(\mathbb{R}^{d}\right)$, the space of globally Lipschitz continuous functions from $\mathbb{R}^{d}$ to $\mathbb{R}^{d}$.

Notice that the interaction kernel satisfies the assumptions (3.6) and (3.7) if and only if

$$
W \in C^{1}\left(\mathbb{R}^{3}\right), \quad \nabla W \in \operatorname{Lip}\left(\mathbb{R}^{3} ; \mathbb{R}^{3}\right) \text { and } \nabla W(z)+\nabla W(-z)=0 \text { for all } z \in \mathbb{R}^{3} .
$$

In order to appreciate the stochastic contribution later, it may be useful to review the role of the above assumptions.

\section{Remark 3.1.}

(i) The assumptions (3.6) and the fact that $K$ is an operator of interaction, allow to suppose that $K\left(z, z^{\prime}\right)=-K\left(z^{\prime}, z\right)$, so that $K(0,0)=0$ (to avoid no self-interaction). These two conditions imply in particular that

$$
\left|K\left(z, z^{\prime}\right)\right| \leq L\left|z-z^{\prime}\right| \leq L\left(|z|+\left|z^{\prime}\right|\right), \quad z, z^{\prime} \in \mathbb{R}^{d}
$$

and ensures that the differential system (1.1) has a global solution, defined for all $t \in \mathbb{R}$.

(ii) The assumption (3.7) implies that $K$ is Lipschitz continuous in $z$, uniformly in $z^{\prime}$. That is:

$$
\left\{\begin{array}{l}
\sup _{z^{\prime} \in \mathbb{R}^{d}}\left|K\left(z_{1}, z^{\prime}\right)-K\left(z_{2}, z^{\prime}\right)\right| \leq L\left|z_{1}-z_{2}\right|, \\
\sup _{z \in \mathbb{R}^{d}}\left|K\left(z, z_{1}\right)-K\left(z, z_{2}\right)\right| \leq L\left|z_{1}-z_{2}\right| .
\end{array}\right.
$$

\subsection{Formal derivation of Eq. (3.1) from (1.1).}

Since all particles are assumed identical, we do not really need to take care about the state of each particle individually: it is sufficient to know the state of the system up to permutation of particles. In slightly pedantic terms, we are taking the quotient of the phase space $\left(\mathbb{R}^{d} \times \mathbb{R}^{d}\right)^{N}$ by the permutation group $\mathfrak{S}_{N}$, thus obtaining a cloud of indistinguishable points.

To each $Z_{N} \in\left(\mathbb{R}^{d}\right)^{N}$ we associate the empirical measure

$$
\mu_{Z_{N}}:=\frac{1}{N} \sum_{k=1}^{N} \delta_{x_{k}} \otimes \delta_{v_{k}} \in \mathscr{P}\left(\mathbb{R}^{d} \times \mathbb{R}^{d}\right)
$$

and, for each $t \in \mathbb{R}$, we define

$$
\mu_{N}^{Z}(t):=\mu_{T_{t}^{N} Z_{N}^{i n}}, \quad t \in \mathbb{R}
$$

where $\delta_{z_{k}}$ is the Dirac mass in the phase space at $(\bar{x}, \bar{v}) \in \mathbb{R}^{2 d}$. From the physical point of view, the empirical measure counts particles in phase space, in other words $\mu_{t}^{N}[A]$ is the proportion of particles in a set $A$ of $\mathbb{R}^{2 d}$ :

$$
\mu_{t}^{N}[A]=\frac{1}{N} \operatorname{card}\left\{i ;\left(x_{i}(t), v_{i}(t)\right) \in A\right\} .
$$

One of the simplest way to understand formally how to derive Eq. (3.1) is to introduce the empirical measure $\mu_{N}^{Z}(t)$. In fact if $\left(X_{i}, V_{i}\right)_{1 \leqslant i \leqslant N}$ is solution to (1.1) and if there is no self-interaction: 
$\nabla \Phi(0)=F(0)=0$, then $\mu_{N}^{Z}$ solves (3.1) in the sense of distribution. Formally one may then expect that any limit of $\mu_{N}^{Z}$ still satisfies the same equation.

In order to consider a larger class of initial conditions, we make precise what we mean by solutions.

Definition 3.1. Let $V \in C\left([0, T] \times \mathbb{R}^{d}\right)$, and let $\mu^{\text {in }} \in \mathscr{M}\left(\mathbb{R}^{d}\right)$. A weak solution of the Cauchy problem for the conservative transport equation

$$
\left\{\begin{array}{l}
\partial_{t} \mu+\operatorname{div}(\mu V)=0 \\
\left.\mu\right|_{t=0}=\mu^{i n}
\end{array}\right.
$$

is an element $\mu \in C\left([0, T] ; w-\mathscr{M}\left(\mathbb{R}^{d}\right)\right)$ that satisfies the initial condition and the equality

$$
\int_{0}^{T} \int_{\mathbb{R}^{d}}\left(\partial_{t} \phi(t, x)+V(t, x) \cdot \nabla_{x} \phi(t, x)\right) \mu(t, d x) d t=0
$$

for any test function $\phi \in C_{c}^{1}\left((0, T) \times \mathbb{R}^{d}\right)$.

Remark 3.2. If $f \in L^{1}\left([0, T) \times \mathbb{R}^{2 d}\right)$ is a weak solution (in the sense of distributions) to (3.1), then $\mu_{t}(d x, d v)=f(x, v, t) d x d v$ is a measure valued solution to (3.1). If $\mu$ is a measure valued solution to (3.1) and $\mu_{t}$ is the absolutely continuous measure with respect to Lebesgue measure whose distribution function is given by $f \in L^{1}\left([0, T) \times \mathbb{R}^{2 d}\right)$, i.e. $\mu_{t}(d x, d v)=f(x, v, t) d x d v$, then $f$ is a distributional weak solution to the (3.1).

By referring to ODE (1.1) the time-dependent empirical measure $\mu_{N}$ is a weak solution to the Vlasov equation (3.1), where the operator $\mathscr{K}$ in (3.1) is extended to $\mathscr{P}_{1}\left(\mathbb{R}^{d}\right)$ by the formula

$$
\mathscr{K} \mu(z)=\int_{\mathbb{R}^{d}} K\left(z, z^{\prime}\right) \mu\left(d z^{\prime}\right) .
$$

This idea goes back at least to $[20,24]$ which consists in representing an $N$-particle configuration $Z_{t}^{N}$ as a sum of Dirac measures

$$
Z_{t}^{N}=\left(Z_{1, t}, \ldots, Z_{N, t}\right) \quad \longleftrightarrow \quad \mu_{Z_{t}^{N}}^{N}=\frac{1}{N} \sum_{j=1}^{N} \delta_{Z_{j, t}} \in \mathscr{P}\left(\mathbb{R}^{d}\right)
$$

and proving that, $\mu_{Z_{t}^{N}}^{N}$ converge, in weak sense, to $f_{t}^{N}$.

The main result of this section follows.

Theorem 3.1. Consider the N-particle evolution model

$$
\left\{\begin{array}{l}
\dot{z}_{i}(t)=\mathbb{G}\left(z_{i}\right)+\frac{1}{N} \sum_{j=1}^{N} K\left(z_{i}(t), z_{j}(t)\right) \\
z_{i}(0)=z_{i}^{i n}
\end{array}\right.
$$

where $i=1, \ldots, N$. 
(i) Assume that the interaction kernel $K \in C^{1}\left(\mathbb{R}^{d} \times \mathbb{R}^{d}, \mathbb{R}^{d}\right)$ satisfies the assumption (3.5) (or possibly (3.6)-(3.10)). Then, the Cauchy problem (3.15) has a unique solution of class $C^{1}$ on $\mathbb{R}$ denoted by

$$
\left(z_{1}(t), \ldots, z_{N}(t)\right)=: T_{t}^{N}\left(z_{1}^{i n} \ldots, z_{N}^{i n}\right)
$$

for all initial data $z_{1}^{i n}, \ldots, z_{N}^{i n} \in \mathbb{R}^{d}$.

(ii) To each $Z_{N} \in\left(\mathbb{R}^{d}\right)^{N}$, introduce an empirical measure defined on the one-particle phase space by

$$
\mu_{N}(d x, d v ; d t):=\mu_{Z_{N}}(x, v)=\frac{1}{N} \sum_{k=1}^{N} \delta_{z_{k}}(x, v)
$$

and, for each $t \in \mathbb{R}$, define

$$
\mu_{N}(t):=\mu_{T_{t}^{N} Z_{N}^{i n}}, \quad t \in \mathbb{R}
$$

If

$$
\lim _{N \rightarrow \infty} \int d \mu_{N}(0 ; z) \varphi(z)=\int f_{0}(z) \varphi(z) d z
$$

for all $\varphi$ continuous and bounded, then for all $t>0$

$$
\lim _{N \rightarrow \infty} \int d \mu_{N}(t ; z) \varphi(z)=\int f(t, z) \varphi(z) d z
$$

where $f(t)$ is solution of the following system

$$
\left\{\begin{array}{l}
\partial_{t} f+\operatorname{div}_{z}(f \mathscr{K} f)=0, \\
f(0, z)=f_{0}(z) .
\end{array}\right.
$$

Before proving this theorem, some comments are in order.

Remark 3.3. Note that, for each $Z_{N}^{i n} \in\left(\mathbb{R}^{d}\right)^{N}$, the time-dependent empirical measure $\mu_{N}$ defined in (3.17) belongs to $C\left(\mathbb{R}_{+}, w-\mathscr{P}_{1}\left(\mathbb{R}^{d}\right)\right)$.

The point ( $\mathrm{ii}$ ) of Theorem 3.1 states that, in terms of the empirical measure $\mu(t)$ of the particles, one then has in the transport PDE (3.20) the continuum counterpart of (3.15). Roughly speaking, Theorem 3.1 says precisely that the solution of the equation (1.4), at any fixed time $t>0$, is continuous with respect to the initial data, for the topology induced by the weak convergence of the measures, expressed by condition (3.18). Since $\mathscr{K}$ si smooth enough, a classical characteristic method can be used to prove existence and uniqueness of solutions in a measure sense. The key to 
our proof is the fact that any positive solution of

$$
\left\{\begin{array}{l}
\partial_{t} \mu_{t}+\operatorname{div}\left(b_{t} \mu_{t}\right)=0 \\
\mu(0, \cdot)=\mu_{0}
\end{array}\right.
$$

is a solution of the associated first-order ordinary differential equation

$$
\dot{\gamma}=b_{t}(\gamma)
$$

For instance, first assume $b_{t}$ to be smooth, and let $Y(s, t, y)$ denote the flow map

$$
\left\{\begin{array}{l}
\frac{d}{d t} Y(t, s, y)=v_{t}(Y(t, s, y)), \\
Y(s, s, x)=y
\end{array}\right.
$$

Then the unique solution of (3.21) is constructed by transporting the initial measures through the empirical measure $\mu_{t}$ and is given by

$$
\mu_{t}=Y(t, 0, \cdot) \# \mu_{0} \quad \forall t \in[0, T],
$$

where \# means the following: $f \# \mu(B):=\mu(f)^{-1}(B)$ for any Borel set.

We now provide the proof of Theorem 3.1.

\section{Proof of Theorem 3.1.}

Since we assume that $\mathbb{G}$ and $K$ are Lipschitz continuous, the existence and uniqueness of the trajectories $z_{i}(t)$ of Eq. (3.15) is guaranteed by the Picard-Lindelöf Theorem for ordinary differential equations.

A strategy to attack the problem (3.20) is to investigate its weak solution. As already mentioned, the nonlinear Vlasov-like equation is a transport equation, and can therefore be solved by the wellknown method of characteristics. It is given by the following results.

Proposition 3.1. Let $b \equiv b(t, y) \in C\left([0, \tau] ; \mathbb{R}^{d}\right)$ be such that $D_{y} b \in C\left([0, \tau] ; \mathbb{R}^{d}\right)$ and $b$ has at most polynomial growth, namely

$$
|b(t, y)| \leqslant \kappa(1+|y|)
$$

for all $t \in[0, \tau]$ and $y \in \mathbb{R}^{d}$, where $\kappa$ is a positive constant. Then

(1) For each $t \in[0, \tau]$, the Cauchy problem for the $O D E$

$$
\left\{\begin{array}{l}
\dot{Y}(s)=b(s, Y(s)), \\
Y(t)=y
\end{array}\right.
$$

has a unique solution $s \mapsto Y(s, t, y)$ and the map $Y$ satisfies

$$
Y\left(t_{3}, t_{2}, Y\left(t_{2}, t_{1}, y\right)\right)=Y\left(t_{3}, t_{1}, y\right),
$$

i.e. the flow satisfies the so-called semigroup property. 
(2) Let $f \in C^{1}\left(\mathbb{R}^{d}\right)$. The Cauchy problem for the transport equation

$$
\left\{\begin{array}{l}
\partial_{t} f(t, y)+b(t, y) \cdot \nabla_{y} f(t, y)=0, \\
f(0, y)=f_{0}(y)
\end{array}\right.
$$

has a unique solution $f \in C^{1}\left([0, \tau] \times \mathbb{R}^{d}\right)$; this solution is given by the formula

$$
f(t, y)=f^{0}(Y(0, t, y)) .
$$

(3) Let $\mu$ be a Borel probability measure on $\mathbb{R}^{d}$. The push-forward measure

$$
\mu(t):=Y(t, 0,) \# \mu^{0}
$$

is a weak solution of

$$
\left\{\begin{array}{l}
\partial_{t} \mu+\operatorname{div}_{y}(\mu b)=0, \\
\left.\mu\right|_{t=0}=\mu^{0}
\end{array}\right.
$$

(4) The unique weak solution $\mu \in C^{1}\left([0, \tau], w-\mathscr{P}\left(\mathbb{R}^{d}\right)\right)$ of the Cauchy problem considered in (3.25) is the push-forward measure defined by the formula

$$
\mu(t):=Y(t, 0,) \# \mu^{0}
$$

for each $t \in[0, \tau]$.

Before proceeding, we draw the attention to some simple observations relating the Proposition 3.1. These comments are intended as further motivation.

\section{Remark 3.4.}

(i) The hypothesis (3.24) ensures that the flow $Y$ is globally defined - that is to say for all $t$ such that the field $b(t, \cdot)$ is defined and $C^{1}$.

(ii) The group property (3.26) guarantees that almost every initial condition $(s, y) \in[0, \tau] \times \mathbb{R}^{d}$ lies on exactly one trajectory.

(iii) The particle dynamics (3.15) induces through $\mu(t)$ a solution (3.25) in a weak form.

(iv) We consequently infer that the trajectories, defined through the ordinary differential equation (3.25), exist $\mu$-a.e. and that for any compactly supported test function $\varphi \in C_{c}\left(\mathbb{R}^{d}\right)$ it holds

$$
\int_{\mathbb{R}^{d}} \varphi d \mu_{t}=\int_{\mathbb{R}^{d}} \varphi(Y(t, y)) d \mu^{0}(y), \quad \forall \varphi \in C_{c}\left(\mathbb{R}^{d}\right) .
$$

The property (3.30) therefore can only interpreted as a very weak notion of solving the continuity equation (3.30).

(v) Notice that the parts (3) and (4) of the Proposition 3.1 do not solve the uniqueness problem, but ensure only that, if one suppresses the coupling between $K$ and $\mu$, there is a unique 
weak measure-valued solution to the transport equation

$$
\left\{\begin{array}{l}
\frac{d X_{t}}{d t}=V_{t} \\
\frac{d V_{t}}{d t}=\mathbb{G}(X)+\int_{\mathbb{R}^{d}} K\left(z, z^{\prime}\right) f\left(t, d z^{\prime}\right) \\
\left(X_{0}, V_{0}\right)=(X, V)
\end{array}\right.
$$

which can be represent with the help of characteristics and can be rewritten as

$$
\frac{d}{d s}(X, V)=\mathbf{b}\left(X_{s}, v_{s}\right)
$$

where the vector field $\mathbf{b}:[0, T] \times \mathbb{R}^{d} \times \mathbb{R}^{d} \rightarrow \mathbb{R}^{d} \times \mathbb{R}^{d}$ of the right-hand side of Eqs. (3.31) is defined by

$$
\mathbf{b}(x, v)=(v, \mathscr{K}[f](x)) .
$$

Let $T_{t}$ be the semigroup of the characteristics related to the vector field (3.33). We thus have

$$
\left(X_{t}, V_{t}\right)=T_{t}\left(X_{0}, V_{0}\right) \text {. }
$$

Then immediately we have

$$
\mu_{T_{t} Z_{N}^{i n}}=T_{t} \# \mu_{0 Z_{N}^{i n}}
$$

\section{Proof of Proposition 3.1.}

The proof of Proposition 3.1 rests solely on the method of characteristics of transport equation, which reduces the study of our PDEs to the study of ODE systems.

(1) A solution to this problem is a function $Y \in C^{1}\left(\left[t_{1}, t_{2}\right] ; \mathbb{R}^{d}\right)$ which is a characteristic curve of $b$ and satisfies the condition $Y\left(t_{0}\right)=y$. In particular we must require $t_{0} \in\left[t_{1}, t_{2}\right]$. Next observe that, since $b$ satisfies (3.24), it satisfies the assumptions of the Cauchy-Lipschitz theorem. Therefore, the differential system of characteristics has a unique $C^{1}$ maximal solution $Y$ that is defined on some interval $I(t, y) \subset[0, \tau]$ such that $s \in I(x, s)$.

For all $t_{1}, t_{2} \in[0, T]$ and $x \in \mathbb{R}^{n}$ the maps

$$
t_{3} \mapsto Y\left(t_{3}, t_{2}, Y\left(t_{2}, t_{1}, y\right)\right) \quad \text { and } \quad t_{3} \mapsto Y\left(t_{3}, t_{1}, y\right)
$$

are two curves integrals of $b$ passing through $Y\left(t_{2}, t_{1}, x\right)$ for $t_{3}=t_{2}$. By uniqueness of CauchyLipschitz theorem, they thus coincide on their maximum interval of definition, i.e. for all $t_{3} \in[0, T]$, which proves the announced equality.

(2) (i) Existence. The function $(t, s) \mapsto f_{0}\left(Y(0, t, y)\right.$ is a composition of the maps $f_{0}$ and $(t, y) \mapsto$ $Y(0, t, y)$ which are both of class $C^{1}$, thus it defines an element of $C^{1}\left([0, T] \times \mathbb{R}^{d}\right)$.

(ii) Uniqueness. If $f \in C\left([0, \tau] \times \mathbb{R}^{d}\right)$, the map

$$
\phi: s \mapsto \phi(s)=f(s, Y(s, 0, y)) \in \mathbb{R}
$$

is of class $C^{1}$ on $[0, \tau]$ being the composition of the $C^{1}$ maps $f$ and $(t, y) \mapsto Y(0, t, y)$. By the chain rule

$$
\frac{d}{d t} f(t, Y(t, 0, y))=\partial_{t} f(t, Y(t, 0, y))+\nabla_{y} f(t, Y(t, 0, y)) \cdot \partial_{s} Y(t, 0, y)
$$




$$
\begin{aligned}
& =\partial_{t} f(t, Y(t, 0, y))+\nabla_{y} f(t, Y(t, 0, y)) \cdot b(t, Y(t, 0, y)) \\
& =\left(\partial_{t} f+b \cdot \nabla_{y} f\right)(t, X(t, 0, y))=0 .
\end{aligned}
$$

An immediate consequence of theses computations is that the map $t \mapsto f(t, Y(t, 0, y))$ is constant on $[0, T]$, so that

$$
f(t, Y(t, 0, y))=f(0, y)=f_{0}(y) .
$$

The change of variable $z:=Y(t, 0, y)$ implies $y=Y(0, t, z)$ so that

$$
f(t, y)=f_{0}(Y(0, t, y)) \text { for each }(t, y) \in[0, T] \times \mathbb{R}^{d} .
$$

(3) Let $\phi \in C_{c}^{1}\left([0, \tau], \mathbb{R}^{d}\right)$. By the part (1) of the proposition, we also can argue that the function

$$
t \mapsto \int_{\mathbb{R}^{n}} \phi(t, Y(t, 0, y)) \mu^{i n}(d y)
$$

is of class $C^{1}$ on $[0, \tau]$, so if one denotes by

$$
\mu(t):=Y(t, 0, \cdot) \# \mu^{i n}
$$

the push-forward measure, one then obtains

$$
\begin{aligned}
\frac{d}{d t} \int_{\mathbb{R}^{n}} \phi(Y(t, 0, y)) \mu^{i n}(d y) & =\int_{\mathbb{R}^{n}}\left(\partial_{t} \phi(t, Y(0, t, y))+\nabla_{x} \phi(t, Y(t, 0, y)) \cdot \partial_{t} Y(t, 0, y) \mu^{i n}(d y)\right. \\
& =\int_{\mathbb{R}^{n}}\left(\partial_{t} \phi(t, Y(0, t, y))+\nabla_{x} \phi(t, Y(t, 0, y)) \cdot b(t, Y(t, 0, y)) \mu^{i n}(d y)\right. \\
& =\int_{R^{n}}\left(\partial_{t} \phi(t, x)+b(t, y) \cdot \nabla_{x} \phi(t, y)\right) \mu(t, d y),
\end{aligned}
$$

after substituting $y$ to $Y(0, t, y)$. Integrating both sides of the equality above with respect to the variable $t$, one finds that

$$
\begin{aligned}
0 & =\left[\int_{\mathbb{R}^{n}} \phi(t, Y(t, 0, y)) \mu^{i n}(d y)\right]_{t=0}^{t=T} \\
& =\int_{0}^{T}\left(\frac{d}{d t} \int_{\mathbb{R}^{n}} \phi(t, Y(t, 0, y)) \mu^{i n}(d y)\right) d t \\
& =\int_{0}^{T} \int_{\mathbb{R}^{n}}\left(\partial_{t} \phi(t, x)+b(t, x) \nabla \phi(t, x)\right) \mu(t, d x) d t .
\end{aligned}
$$

The above calculation indicates that $\mu$ is a weak solution of the transport equation. Since it obviously satisfies the initial condition, $\mu$ is a weak solution of the Cauchy problem.

(4) To prove that the unique weak solution $\mu \in C\left([0, \tau], w-\mathscr{P}\left(\mathbb{R}^{d}\right)\right)$ of the Cauchy problem considered in (3.25) is the push-forward measure defined by the formula $\mu(t):=Y(t, 0, \cdot) \# \mu^{i n}$ 
for each $t \in[0, \tau]$, we compute, for $\phi \in C_{c}^{1}\left(\mathbb{R}^{d}\right)$,

$$
\frac{d}{d t}\langle Y(0, t, \cdot) \# \mu(t), \phi\rangle \in \mathscr{D}^{\prime}([0, \tau]) .
$$

Let us define the function $\left.\chi \in C_{c}^{1}[0, \tau]\right)$ and let us introduce the following notation

$$
v(t):=Y(0, t, \cdot) \# \mu(t) .
$$

We claim that

$$
-\int_{0}^{\tau} \chi^{\prime}(t)\left(\int_{\mathbb{R}^{d}} \psi(x) v(t, d y)\right) d t=-\int_{0}^{\tau} \int_{\mathbb{R}^{d}} \chi^{\prime}(t) \psi(Y(0, t, y)) \mu(t, d y) d t .
$$

By virtue of the statement (3) of the Theorem, we know that the map $(t, x) \mapsto \psi(Y(0, t, y))$ is of class $C^{1}$ on $[0, \tau] \times \mathbb{R}^{d}$ and satisfies

$$
\left(\partial_{t}+b(t, x) \nabla_{y} \psi(Y(0, t, y))=0, \quad \forall(t, x) \in[0, \tau] \times \mathbb{R}^{d} .\right.
$$

The function $\psi(t, y)$ defined by $\Psi(t, y)=\chi(t) \psi(Y(0, t, y))$ is of class $C^{1}\left([0, T] \times \mathbb{R}^{d}\right)$ and satisfies

$$
\left(\partial_{t}+b(t, x) \nabla_{y} \Psi(Y(0, t, y))=\chi^{\prime}(t) \psi(Y(0, t, y)), \quad \forall(t, x) \in[0, \tau] \times \mathbb{R}^{d} .\right.
$$

These arguments suggest that if we knew that $\operatorname{supp}(\Psi)$ is compact in $[0, \tau] \times \mathbb{R}^{d}$, since $\mu$ is a weak solution of the transport equation, we could deduce that

$$
-\int_{0}^{\tau} \chi^{\prime}(t)\left(\int_{\mathbb{R}^{d}} \psi(x) v(t, d x)\right) d t=-\int_{0}^{\tau}\left(\partial_{t}+b(t, x) \nabla_{y} \Psi(t, y) \mu(t, d y) d t=0\right.
$$

and the continuous function

$$
h: t \mapsto \int_{\mathbb{R}^{d}} \psi(x) v(t, d x)
$$

satisfies

$$
\frac{d}{d t} \int_{\mathbb{R}^{d}} \psi(x) v(t, d x)=0 \quad \text { in } \mathscr{D}^{\prime}((0, \tau)) .
$$

Based on this smoothing, we deduce that this function is constant on $[0, \tau]$. Hence, by applying the above argument, we get

$$
\int_{\mathbb{R}^{d}} \psi(x) v(t, d x)=\int_{\mathbb{R}^{n}} \psi(x) v(0, d x)=\int_{\mathbb{R}^{d}} \psi(0, d x)=\int_{\mathbb{R}^{n}} \psi(x) \mu^{i n}(d x) .
$$

Since this identity holds for each $\psi \in C_{c}^{1}\left(\mathbb{R}^{d}\right)$, we conclude that

$$
v=Y(0, t, \cdot) \# \mu(t)=\mu^{i n},
$$

whereby

$$
\mu=Y(0, t, \cdot) \# \mu(t)=\mu^{i n}, \quad \text { for all } t \in[0, \tau]
$$


It remains to prove that the support of $\Psi$ is compact in $[0, \tau] \times \mathbb{R}^{d}$. The hypothesis (3.24) on the vector field $b$ implies that

$$
|Y(s, t, y)| \leqslant(|y|+\kappa \tau) e^{\kappa \tau}, \quad \forall t \in \mathbb{R}^{d}, s, t, \in[0, \tau] .
$$

This inequality ensures that

$$
\operatorname{supp}(\psi) \subset B(0, R) \Rightarrow \operatorname{supp}(\psi \circ Y(0, t, \cdot)) \subset B\left(0,(R+\kappa \tau) e^{\kappa \tau}\right),
$$

for all $t \in[0, \tau]$. Since $\chi$ has support in $[\varepsilon, \tau-\varepsilon]$ for some $\varepsilon>0$, we deduce that

$$
\operatorname{supp}(\Psi) \subset[\varepsilon, \tau-\varepsilon] \times B\left(0,(R+\kappa \tau) e^{\kappa \tau}\right) .
$$

We have thereby established assertion of the compacity of the support of $\Psi$ completing the proof of the proposition 3.1.

\section{Mean-field characteristic flow}

Let us pass now to analyze another important feature of the Eq. (3.15), that is how the mean field characteristic flow $Z$ and the flow $T_{t}$ are related to the $N$-particle ODE system. Roughly speaking, the equation of mean field characteristic derived from (1.1) admits a mean field characteristic flow. The existence and uniqueness of the solution of (3.1) will be obtain as a consequence of the construction of the mean field flow. We will prove that there exists a unique map

$$
\mathbb{R}^{+} \times \mathbb{R}^{2 d} \times \mathscr{P}_{1}\left(\mathbb{R}^{d}\right) \ni\left(t, z^{i n}, \mu^{i n}\right) \mapsto Z\left(t, z^{i n}, \mu^{i n}\right) \in \mathbb{R}^{2 d}
$$

such that $t \mapsto Z\left(t, z^{i n}, \mu^{i n}\right)$ is the integral curve of the vector field

$$
z \mapsto \int_{\mathbb{R}^{2 d}} K\left(z, z^{\prime}\right) \mu\left(t, d z^{\prime}\right)=:(\mathscr{K} \mu(t))(z)
$$

passing through $z^{i n}$ at time $t=0$, where $\mu(t):=Z\left(t, \cdot, \mu^{i n}\right) \# \mu^{i n}$.

Assume that $f_{0}$ is a probability density on $\mathbb{R}^{3} \times \mathbb{R}^{3}$. Applying the method of characteristics to the transport equation governing $f$ shows that

$$
f\left(t, X\left(t, x, v ; f_{0}\right), V\left(t, x, v ; f_{0}\right)\right)=f_{0}(x, v)
$$

where $t \mapsto(X, V)\left(t, \cdot, \cdot ; f_{0}\right)$ is the solution of (3.31).

Since $\operatorname{div}_{x, v}\left(V, \mathbb{G}(X)+\int_{\mathbb{R}^{d}} K\left(z, z^{\prime}\right) f\left(t, d z^{\prime}\right)\right)=0$, the flow $(X, V)$ leaves the Lebesgue measure $d x d v$ invariant so that (4.1) can be recast as

$$
f(t, \cdot, \cdot) d x d v=(X, V)\left(t, \cdot, \cdot ; f_{0}\right) \# f_{0} d X d V .
$$

Therefore the characteristic flow $Z:=(X, V)$ of the Vlasov-type equation (3.1) satisfies a mean-field integro-differential system of the form

$$
\left\{\begin{array}{l}
\dot{Z}=\mathscr{K} \mu(t, Z) \\
\mu(t, \cdot)=Z\left(t, \cdot ; \mu^{i n}\right) \# \mu^{i n} \\
Z\left(0, z ; \mu^{i n}\right)=z
\end{array}\right.
$$

Our result is stated as follows. 
Proposition 4.1. For each Borel probability measure $\mu^{\text {in }}$ on $\mathbb{R}^{d}, Z \equiv Z\left(t, z ; \mu^{i n}\right)$ is the solution of the mean-field integro-differential.

Then, there exists a unique global solution $Z \in C\left(\mathbb{R}_{+} \times \mathbb{R}^{d} ; \mathbb{R}^{d}\right)$ to (4.3) such that $t \mapsto Z(t, z)$ is continuously differentiable on $\mathbb{R}_{+}$for each $z \in \mathbb{R}^{d}$.

\section{Proof of Proposition 4.1.}

The proof falls in two major steps. The first step consist in establishing the estimate on the operator $K$ by rewriting the differential equation (4.3) as an integral equation for the unknown function $Z$. The second major steps is to use the integral equation to construct a sequence of functions $\left\{Z_{n}\right\}$ and then showing that this sequence has a unique limit as $n \rightarrow \infty$. This limiting function is the solution of the original initial value problem.

- 1st Step. We express (4.3) into the form

$$
\left\{\begin{array}{l}
\partial_{t} Z(t, \zeta)=G(z)+\int_{\mathbb{R}^{d}} K\left(z(t, \zeta), Z\left(t, \zeta^{\prime}\right)\right) \mu^{i n}\left(d \zeta^{\prime}\right) \\
Z(0, \zeta)=\zeta
\end{array}\right.
$$

and get the estimate on the map

$$
s \mapsto G(z)+\int_{\mathbb{R}^{d}} K\left(Z(s, \zeta), Z\left(s, \zeta^{\prime}\right)\right) \mu^{i n}\left(d \zeta^{\prime}\right)
$$

To this end, one defines the Banach space

$$
X:=\left\{v \in C\left(\mathbb{R}^{d} ; \mathbb{R}^{d}\right) \text { s.t. } \sup _{z \in \mathbb{R}^{d}} \frac{|v(z)|}{1+|z|}<\infty, \quad\|v\|_{X}:=\sup _{z \in \mathbb{R}^{d}} \frac{|v(z)|}{1+|z|}\right\} .
$$

Thanks to the hypothesis (3.7), for $v, w \in X$, one has

$$
\left|\int_{\mathbb{R}^{d}} K\left(v(z), v\left(z^{\prime}\right)\right) \mu^{i n}\left(d z^{\prime}\right)-\int_{\mathbb{R}^{d}} K\left(w(z), w\left(z^{\prime}\right)\right) \mu^{i n}\left(d z^{\prime}\right)\right| \leqslant L\left(2+C_{1}\right)(1+|z|)\|v-w\|_{X} .
$$

where $C_{1}$ is a positive constant.

- 2nd Step. With this estimate, we construct the solution by the usual Picard iteration procedure. Define recursively a sequence of maps $\left(Z^{n}\right)_{n \geq 0}$ by

$$
\left\{\begin{array}{l}
Z^{n+1}(t, z):=z+\int_{0}^{t} K\left(Z^{n}(\cdot, \cdot) \# \mu^{i n}\right)\left(\tau, Z^{n}(\tau, z)\right) d \tau+\mathbb{G}(z), \quad t \geq 0, \\
Z^{0}(t, z):=z .
\end{array}\right.
$$

Applying (4.5) iteratively, and notice that

$$
\int \mathbf{1}_{0 \leq t_{n} \leq t_{n-1} \leq \ldots \leq t_{1} \leq t} d t_{n} d t_{n-1} \ldots d t_{1}=\frac{t^{n}}{n !}
$$

one checks that

$$
\left\|Z^{n+1}(t, \cdot)-Z^{n}(t, \cdot)\right\|_{X} \leqslant\|G\|_{X}+\frac{\left(\left(2+C_{1}\right) L|t|\right)^{n+1}}{n !} .
$$


An immediate consequence of the (4.6) is that the sequence $Z^{n}$ converges uniformly on $[0, T] \times \mathbb{R}^{d}$ for each $T>0$ towards a solution of integral equation

$$
Z(t, z)=z+\int_{0}^{t} K\left(Z(\cdot, \cdot) \# \mu^{i n}\right)(\tau, Z(\tau, z)) d \tau+\mathbb{G}(z),
$$

or, equivalently, to

$$
Z(t, \zeta)=\zeta+\int_{0}^{t} \int_{0}^{t} K\left(Z(s, \zeta), Z\left(s, \zeta^{\prime}\right)\right) \mu^{i n}\left(d \zeta^{\prime}\right)+\mathbb{G}\left(Z\left(s, \zeta^{\prime}\right)\right) d s,
$$

defined for each $t \geq 0$ and such that $Z \in C\left(\mathbb{R}_{+} \times \mathbb{R}^{d} ; \mathbb{R}^{d}\right)$. If $Z_{1}$ and $Z_{2} \in C(\mathbb{R}, X)$ are two solutions of the integral equation (4.8), one has

$$
\begin{aligned}
Z_{1}(t, \zeta)-Z_{2}(t, \zeta)=\int_{0}^{t} K & \left(Z_{1}(s, \zeta), Z_{1}\left(s, \zeta^{\prime}\right)\right) \int_{0}^{t} K\left(Z_{2}(s, \zeta), Z_{2}\left(s, \zeta^{\prime}\right)\right) \mu^{i n}\left(d \zeta^{\prime}\right) \\
& +\left(\mathbb{G}_{1}\left(Z_{1}\left(s, \zeta^{\prime}\right)\right)-\mathbb{G}_{2}\left(Z_{2}\left(s, \zeta^{\prime}\right)\right) .\right.
\end{aligned}
$$

By straightforward estimation, it follows that, for all $t \in \mathbb{R}$

$$
\left\|Z_{1}(t, \cdot)-Z_{2}(t, \cdot)\right\|_{X} \leqslant L\left(2+C_{1}\right)\left|\int_{0}^{t}\left\|Z_{1}(s, \cdot)-Z_{2}(s, \cdot)\right\|_{X} d s\right|+\left\|\mathbb{G}_{1}(s, \cdot)-\mathbb{G}_{2}(s, \cdot)\right\|_{X} .
$$

To conclude, we observe that from the bound (4.9), we have that

$$
\left\|Z_{1}(t, \cdot)-Z_{2}(t, \cdot)\right\|_{X}=0
$$

by Gronwall's inequality, so that $Z_{1}=Z_{2}$. Then immediately implies that the integral equation has only one solution $Z \in C(\mathbb{R}, ; X)$ and then proof of Proposition 4.1 is gained.

\section{Stability result in bounded Lipschitz distance}

In this section, we derive a stability estimate for measure valued solution to (3.1). Following Dobrušin 1979 [12] (see also Braun and Hepp 1977 [7], and Neunzert 1984 [25]), we have the following.

Proposition 5.1. Let $\mu \equiv \mu(t, d z)$ and $v \equiv v(t, d z)$ be two solutions of (3.1) in $C\left(\mathbb{R}^{+}, w^{*}-\right.$ $\left.\mathscr{M}^{1}\left(\mathbb{R}^{d}\right)\right)$. Then

$$
W(\mu(t), v(t)) \leqslant W\left(\mu^{0}, v^{0}\right) e^{2 t C(\mathbb{G}, K)}, \quad t \geqslant 0
$$

where $C(\mathbb{G}, K)$ is a constant depending on $\mathbb{G}$ and $K$.

The stability result announced in Proposition 5.1 is obtained by using the Monge-KantorovichRubinstein-Wasserstein distance.

\section{Proof of Proposition 5.1.}

To any bounded map $Z \in C\left(\mathbb{R}_{+} \times \mathbb{R}^{d} ; \mathbb{R}^{d}\right)$, associate the map $\tilde{Z} \in C\left(\mathbb{R}_{+} \times \mathbb{R}^{d} ; \mathbb{R}^{d}\right)$ defined by

$$
\tilde{Z}(t, z)=z+\int_{0}^{t} K\left(Z(\cdot, \cdot) \# \rho^{i n}\right)(\tau, Z(\tau, z)) d \tau
$$

Given $\mu, v \in \mathscr{P}_{r}\left(\mathbb{R}^{d}\right)$, we define $\pi(\mu, v)$ to be the set of Borel probability measures on $\mathbb{R}^{d} \times \mathbb{R}^{d}$, with the first and second marginals $\mu$ and $v$ respectively. 
In our setting, the related characteristic equation to (3.1) is written as

$$
\begin{aligned}
& \dot{Z}\left(t, a, \mu^{i n}\right)=\int K\left(Z\left(t, a, \mu^{i n}\right)-\left(Z\left(t, a, \mu^{i n}\right)\right) d \mu^{i n}\left(d a^{\prime}\right)+\mathbb{G}(a)\right. \\
& Z\left(0, a, \mu^{i n}\right)=a
\end{aligned}
$$

which we now express as

$$
\begin{aligned}
Z\left(t, a, \mu^{i n}\right) & =a+\int_{0}^{t} \int K\left(Z\left(t, a, \mu^{i n}\right)-\left(Z\left(t, a, \mu^{i n}\right)\right) \mu^{i n}\left(d a^{\prime}\right)+\mathbb{G}\left(Z\left(s, a, \mu^{i n}\right)\right) d s\right. \\
& =a+\int_{0}^{t} \int K\left(Z\left(t, a, \mu^{i n}\right)-\left(Z\left(t, a, \mu^{i n}\right)\right) \mu^{i n}\left(d a^{\prime}\right)+\mathbb{G}\left(Z\left(s, a, \mu^{i n}\right)\right) d s .\right.
\end{aligned}
$$

Similarly we have

$$
\begin{aligned}
Z\left(t, b, \mu^{i n}\right) & =b+\int_{0}^{t} \int K\left(Z\left(t, a, v^{i n}\right)-\left(Z\left(t, a, v^{i n}\right)\right) v^{i n}\left(d a^{\prime}\right)+\mathbb{G}\left(Z\left(s, b, \mu^{i n}\right)\right) d s\right. \\
& =b+\int_{0}^{t} \int K\left(Z\left(t, a, \mu^{i n}\right)-\left(Z\left(t, a, v^{i n}\right)\right) v^{i n}\left(d a^{\prime}\right)+\mathbb{G}\left(Z\left(s, a, v^{i n}\right)\right) d s .\right.
\end{aligned}
$$

It follows

$$
\begin{aligned}
& \left|Z\left(t, a, v^{i n}\right)-Z\left(t, b, \mu^{i n}\right)\right| \leqslant|a-b| \\
& \quad+\int_{0}^{t} \iint\|K\|_{\text {Lip }}\left|Z\left(s, a, \mu^{i n}\right)-Z\left(s, a^{\prime}, \mu^{i n}\right)-Z\left(s, b, v^{i n}\right)+Z\left(s, b^{\prime}, v^{i n}\right)\right| \pi\left(d a^{\prime} d b^{\prime}\right) d s \\
& \quad+\int_{0}^{t}\|\mathbb{G}\|_{\text {Lip }} Z Z\left(s, a, \mu^{i n}\right)-Z\left(s, b, v^{i n}\right) \mid d s \\
& \leqslant|a-b| \\
& \quad+\int_{0}^{t} \iint\|K\|_{\text {Lip }}\left|Z\left(s, a, \mu^{i n}\right)-Z\left(s, b^{\prime}, v^{i n}\right)\right| \pi\left(d a^{\prime} d b^{\prime}\right) d s+\left(\|K\|_{\text {Lip }}+\|\mathbb{G}\|_{\text {Lip }}\right) \\
& \quad \times \int_{0}^{t}\left|Z\left(s, a, \mu^{i n}\right)-Z\left(s, b^{\prime}, v^{i n}\right)\right| d s .
\end{aligned}
$$

Integrating both sides with respect to $\pi(d a d b)$ yields:

$$
\begin{aligned}
& \iint\left|Z\left(t, a, v^{i n}\right)-Z\left(t, b, \mu^{i n}\right)\right| \leqslant \iint|a-b| \pi(d a d b) \\
& \quad+\int_{0}^{t} \iiint \int\|K\|_{\text {Lip }}\left|Z\left(s, a, \mu^{i n}\right)-Z\left(s, b^{\prime}, v^{i n}\right)\right| \pi\left(d a^{\prime} d b^{\prime}\right) \pi(d a d b) d s \\
& \quad+\left(\|K\|_{\text {Lip }}+\|\mathbb{G}\|_{\text {Lip }}\right) \int_{0}^{t} \iint\left|Z\left(s, a, \mu^{i n}\right)-Z\left(s, b^{\prime}, v^{i n}\right)\right| \pi(d a d b) d s
\end{aligned}
$$

which implies

$$
\begin{aligned}
& \iint\left|Z\left(t, a, v^{i n}\right)-Z\left(t, b, \mu^{i n}\right)\right| \leqslant \iint|a-b| \pi(d a d b) \\
& \quad+\int_{0}^{t} \iint\|K\|_{\text {Lip }}\left|Z\left(s, a, \mu^{i n}\right)-Z\left(s, b^{\prime}, v^{i n}\right)\right| \pi\left(d a^{\prime} d b^{\prime}\right) \pi(d a d b) d s \\
& \quad+\left(\|K\|_{\text {Lip }}+\|\mathbb{G}\|_{\text {Lip }}\right) \int_{0}^{t} \iint\left|Z\left(s, a, \mu^{i n}\right)-Z\left(s, b^{\prime}, v^{i n}\right)\right| \pi(d a d b) d s
\end{aligned}
$$


Equipped with the previous estimates, we deduce

$$
\begin{aligned}
& \iint\left|Z\left(t, a, v^{i n}\right)-Z\left(t, b, \mu^{i n}\right)\right| \leqslant \iint|a-b| \pi(d a d b) \\
& \quad+2\left(\|K\|_{\text {Lip }}+\|\mathbb{G}\|_{\text {Lip }}\right) \int_{0}^{t} \iint\left|Z\left(s, a, \mu^{i n}\right)-Z\left(s, b^{\prime}, v^{i n}\right)\right| \pi(d a d b) d s .
\end{aligned}
$$

Set

$$
\Phi(t)=\iint|a-b| \pi(t, d a d b)=\iint\left|Z\left(t, a, v^{i n}\right)-Z\left(t, b, \mu^{i n}\right)\right|
$$

we get

$$
\Phi(t)=\Phi(0)+2\left(\|K\|_{\text {Lip }}+\|\mathbb{G}\|_{\text {Lip }}\right) \int_{0}^{t} \Phi(s) d s
$$

Rewriting the inequality (5.3) slightly, we can apply Gronwall's lemma to obtain

$$
\Phi(t) \leqslant \Phi(0) e^{2 t\left(\|F\|_{\text {Lip }}+\|\mathbb{G}\|_{\text {Lip }}\right)}
$$

Taking the infinimum on both sides over $\pi\left(\mu^{i n}, v^{i n}\right)$ leads to the desired inequality.

\section{The stochastic evolution case}

For simplicity we assume that all our processes and random variables are defined on some common probability space $\left(\Omega, \mathscr{F},\left(\mathscr{F}_{t}\right)_{0 \leqslant t \leqslant T}, \mathbf{P}\right)$. The formulation we have introduced up to now is motivated by the fact that the equation (1.1) can be interpreted as the evolution equation for a probability density associated to the diffusion process $\left(X_{t}, V_{t}\right) \in \mathbb{R}^{2 d}$, initially distributed according to the density function $f_{0}$ of Eq. (1.1). We will see how our nonlinear problem can be seen as the limit for a linear system of particles, with weak, mean-field interaction.

We denote by $P_{t}$ the measure on $\mathbb{R}^{d}$ with density $f_{t}:=f(t, \cdot)$. We prove here a propagation of chaos result, meaning that the distribution of every fixed $k$-particle subsystem of the $N$-particle system converges when $N$ tends to infinity the to $k$-product $P_{t}^{\otimes k}$ of a probability measure $P_{t}$ defined on the path space (one says that the law of the $N$-particle system is $P$-chaotic). This type of asymptotic behavior beginning to be well known for systems of diffusion processes (see, for example, [30]), and in exchangeable cases it is equivalent to the convergence in law of the empirical measure of the system to $P$.

Our starting point is the following stochastic system of differential equations in $\left(\mathbb{R}^{2 d}\right)^{N}$ :

$$
\left\{\begin{array}{l}
d X_{t}^{(i, N)}=V_{t}^{i, N} d t \\
d V_{t}^{i, N}=\mathbb{G}\left(X_{t}^{(i, N)}, V_{t}^{i, N}\right) d t+\frac{1}{N} \sum_{j=1}^{N} K\left(Z_{t}^{(i, N)}, Z_{t}^{j, N}\right) d t+\sigma d B_{i}^{t}
\end{array}\right.
$$

We shall show that the following statements hold: 
(1) For each $t \geqslant 0$ the empirical measure

$$
\mu_{t}^{N}=\frac{1}{N} \sum_{i=1}^{N} \delta_{\left(X_{t}^{i, N}, V_{t}^{i, N}\right)}
$$

of the particle system converges weakly to a deterministic measure $P_{t}$ on $\mathbb{R}^{2 d}$. This measure has a density $f_{t}:=f(t, \cdot)$ which solves the equation

$$
\partial_{t} f_{t}+v \cdot \nabla_{\mathbf{x}} f_{t}+\operatorname{div}_{v} \cdot\left(\left(\mathbb{G}+\mathscr{K} f_{t}\right) f_{t}\right)=\sigma^{2} \Delta_{v} f_{t}, \quad t>0, x, v \in \mathbb{R}^{d}
$$

where $f_{t}$ is the density of the law at time $t$ of the $\mathbb{R}^{2 d}$-valued process $\left(X_{t}, V_{t}\right)_{t \geqslant 0}$ evolving according to the mean field stochastic differential equation (diffusive Newton's equations):

$$
\left\{\begin{array}{l}
d X_{t}=V_{t} d t \\
d V_{t}=\mathbb{G}\left(X_{t}, V_{t}\right) d t+\left(\mathscr{K} f_{t}(z)\right)\left(X_{t}, V_{t}\right) d t+\sigma d B_{t}^{i}
\end{array}\right.
$$

where

$$
\mathscr{K} \phi(z):=\int_{\mathbb{R}^{d}} K\left(z, z^{\prime}\right) \phi\left(z^{\prime}\right) d z^{\prime}, \quad \phi \in L^{1}\left(\mathbb{R}^{d} ;(1+|z|) d z\right) .
$$

Here $\left(B_{t}^{i}\right)_{t \geqslant 0}$ is a Brownian motion in the velocity space $\mathbb{R}^{d}$ and $f_{t}$ is the law of $\left(X_{t}, V\right)$ in $\mathbb{R}^{2 d}$. Let us note that the stochastic differential equation (6.2) is degenerated insofar as the diffusion acts only on the variable speed.

(2) The distribution of the position $X^{i, N}(t), V^{i, N}(t)$ of each particle also converges weakly to $P_{t}$.

(3) Any fixed number of particles remains approximately independent in the course of time, in spite of the interaction. This statement is know as propagation of chaos.

The particle approximation of solutions to (3.1) consists in the introduction of a large number $N$ of $\mathbb{R}^{2 d}$-valued processes $\left(X_{t}^{N, N}, V_{t}^{N, N}\right) \in \mathbb{R}^{2 d}$ with $1 \leqslant i \leqslant N$, no more evolving by the empirical measure $\mu_{t}^{N}=\frac{1}{N} \sum_{i=1}^{N} \delta_{\left(X_{t}^{i, N} V_{t}^{1, N}\right)}$ of the system, where $X_{t}^{i, N}$ and $V_{t}^{i, N}$ should be understood as the phase at time $t$ of the particle $i$. We let $\left(X_{t}^{(N)} V_{t}^{(N)}\right)_{t \geqslant 0}$ be the solution of the following stochastic system of differential equations in $\left(\mathbb{R}^{2 d}\right)^{N}$ :

$$
\left\{\begin{array}{l}
d X_{t}^{(i, N)}=V_{t}^{i, N} d t \\
d V_{t}^{i, N}=\mathbb{G}\left(X_{t}^{(i, N)}, V_{t}^{i, N}\right) d t+\frac{1}{N} \sum_{j=1}^{N} K\left(Z_{t}^{(i, N)}, Z_{t}^{j, N}\right) d t+\sigma d B_{i}^{t}
\end{array}\right.
$$

Roughly speaking, we will measure in quadratic Monge-Kantorovich-Wasserstein distance, the propagation of chaos building a new particle system and then we show that the propagation of chaos for this new system is uniform in time. In order to state this property we let, for each $i \geqslant 1$, 
$\left(\bar{X}_{t}^{(N)} \bar{V}_{t}^{(N)}\right)_{t \geqslant 0}$ be the solution of the following Vlasov type equation on $\mathbb{R}^{2 d}$ :

$$
\left\{\begin{array}{l}
d \bar{X}_{t}^{i}=\bar{V}_{t}^{i} d t \\
d \bar{V}_{t}^{i}=\mathbb{G}\left(\bar{X}_{t}^{i}, \bar{V}_{t}^{i}\right) d t+\left(\mathscr{K} f_{t}(z)\right)\left(\bar{X}_{t}^{i}, \bar{V}_{t}^{i}\right) d t+\sigma d B_{i}^{i} \\
\left(\bar{X}_{t}^{i}, \bar{V}_{t}^{i}\right) \sim f_{t} .
\end{array}\right.
$$

Let us assume now that particles $i$ in both process $X_{t}^{i}$ and $\bar{X}_{t}^{i}$ are driven by the same Brownian motion $B_{t}^{i}$. This assumption is allowed because by the Lipschitz continuity of functions $(x, v) \mapsto G(x, v)$, $\left(z, z^{\prime}\right) \mapsto K\left(z, z^{\prime}\right)$ both systems (6.4) and (6.5) have a unique strong solution. Moreover, we assume

$$
\bar{Z}_{0}^{i}=Z_{0}^{i}, \quad i=1,2, \ldots n \quad \text { a.s },
$$

and $f_{t}$ is the solution of equation (3.1) associated to initial condition $f_{0}$. The objective is to improve that the limit of the system (6.4), when $N \rightarrow \infty$, is solution the coupled nonlinear SDE (6.5).

The following theorem asserts that, as $N$ goes to infinity, each $X^{i, N}$ has a natural limit $\left(\bar{X}_{t}^{i}, \bar{V}_{t}^{i}\right)$, where $\left(\bar{X}_{t}^{i}, \bar{V}_{t}^{i}\right)$ are independent copies of the solutions of the nonlinear equation (6.5).

Theorem 6.1. (Propagation of chaos) Let $\mathbb{G}$ and $K$ satisfies the Lipschitz conditions and are bounded on $\mathbb{R}^{2 d}$. Let $\left(X_{t}^{i}, V_{t}^{i}\right)$ for $1 \leqslant i \leqslant N$ be the solution for $t$ of the SDE (6.4) for the initial condition $\left(X_{i}^{0}, V_{i}^{0}\right)$ and $\left(\bar{X}_{t}^{i}, \bar{V}_{t}^{i}\right)$ a vector of the solution of the law $f_{t}$ of the $\operatorname{SDE}(6.5)$ for $\left(X_{i}^{0}, V_{i}^{0}\right)$. If $f_{0} \in \mathscr{P}_{2}\left(\mathbb{R}^{2 d}\right)$, then, (6.4), (6.5) and (6.2) are well-posed, and for all $T \geqslant 0$, there exists a constant $C(t)$ depending only in $t$ such that

$$
\sup _{t \in[0, T]} \mathbb{E}\left[\sup _{0 \leqslant s \leqslant t}\left|\left(X_{s}^{i}, V_{s}^{i}\right)-\left(\bar{X}_{s}^{i}, \bar{V}_{s}^{i}\right)\right|\right] \leqslant \frac{C(t)}{\sqrt{N}}
$$

where $|\cdot|$ denotes the Euclidean norm on $\mathbb{R}^{d}$ and $\mathscr{P}_{p}\left(\mathbb{R}^{d}\right)$ is the set of Borel probability measure $\mu$ on $\mathbb{R}^{m}$ with finite moment $\int_{\mathbb{R}^{2 d}}|z|^{p} d \mu(z)$ of order $p$.

Remark 6.1. In particular, the law $f_{t}^{1, N}$ of the couple $\left(X_{t}^{1, N}, V_{t}^{1, N}\right)$ converges towards the law $f_{t}$ of the nonlinear process $\left(\bar{X}_{t}, \bar{V}_{t}\right)$ solution of (6.4) when $N \rightarrow \infty$ since

$$
W_{2}\left(f_{t}^{1, N}, f_{t}\right) \leqslant \mathbb{E}\left[\left|\left(X_{s}^{i}, V_{s}^{i}\right)-\left(\bar{X}_{s}^{i}, \bar{V}_{s}^{i}\right)\right|\right] \leqslant \frac{C(t)}{\sqrt{N}} .
$$

This kind of result is not new, see [30], chapter I.1, Theorem 1.4. But as we have to know exactly the convergence in $N$ of the law at time $t$ of any of the processes $\left(X_{t}^{i}, V_{t}^{i}\right)_{t \geqslant 0}$ towards $f_{t}$, we present the proof.

Proof of Theorem 6.1. We use the techniques used in $[29,30]$ to show propagation of chaos. In order to simplify the notation we omit the indices $N$. For $t \in[0, T]$, we set

$$
\Phi(t):=\mathbb{E}\left[\sup _{0 \leqslant s \leqslant t}\left|\left(X_{s}^{i}, V_{s}^{i}\right)-\left(\bar{X}_{s}^{i}, \bar{V}_{s}^{i}\right)\right|\right] .
$$


Because of the symmetry of the particle system and the system of the nonlinear processes, $\Phi(t)$ does not depend on $i$. By (6.4) and (6.5) we have

$$
\left\{\begin{aligned}
X_{t}-\bar{X}_{t}^{i} & =\int_{0}^{t}\left(V_{s}^{i}-\bar{V}_{s}^{i}\right) d s \\
V_{t}^{i}-\bar{V}_{t}^{i}=\int_{0}^{t}( & \left(\mathbb{G}\left(X_{s}^{i}, V_{s}^{i}\right)-\mathbb{G}\left(\bar{X}_{s}^{i}, \bar{V}_{s}^{i}\right)\right) \\
& \left.\quad+\frac{1}{N} \sum_{j=1}^{N} K\left(Z_{s}^{i}, Z_{s}^{j}\right)-\int_{\mathbb{R}^{d} \times \mathbb{R}^{d}} K\left(z^{i}, y\right) \phi_{s}(y, w) d y d w\right) d s .
\end{aligned}\right.
$$

On the one hand, one has

$$
\mathbb{G}\left(X_{s}^{i}, V_{s}^{i}\right)-\mathbb{G}\left(\bar{X}_{s}^{i}, \bar{V}_{s}^{i}\right) \leqslant[G]_{1}\left|Z_{s}^{i}-\bar{Z}_{s}^{i}\right|^{2} \leqslant C\left|Z_{s}^{i}-\bar{Z}_{s}^{i}\right|^{2},
$$

where $[\varphi]_{1}$ is the Lipschitz seminorm of $\varphi$ on $\mathbb{R}^{2 d}$.

On the other hand, one get

$$
\begin{aligned}
& \left.\frac{1}{N} \sum_{j=1}^{N} K\left(Z_{s}^{i}, Z_{s}^{j}\right)-\int_{\mathbb{R}^{d} \times \mathbb{R}^{d}} K\left(z_{i}, y\right) \phi_{s}(y, w)\right) d s \\
= & \left.\frac{1}{N} \sum_{j=1}^{N}\left(K\left(Z_{s}^{i}, Z_{s}^{j}\right)\right)-K\left(\bar{Z}_{s}^{i}, Z_{s}^{j}\right)\right)+\left(K\left(\bar{Z}_{s}^{i}, Z_{s}^{j}\right)-K\left(\bar{Z}_{s}^{i}, \bar{Z}_{s}^{j}\right)+b_{s}\left(\bar{Z}_{s}^{i}, \bar{Z}_{s}^{j}\right)\right)
\end{aligned}
$$

where

$$
b_{s}\left(x, x^{\prime}\right)=K\left(z, z^{\prime}\right)-\int K(z, y) u_{s}(y, w) .
$$

The Lipschitz continuity of the function $K$ implies that

$$
\begin{aligned}
\left|\frac{1}{N} \sum_{j=1}^{N} K\left(Z_{s}^{i}, Z_{s}^{j}\right)-\int_{\mathbb{R}^{d} \times \mathbb{R}^{d}} K\left(z_{i}, y\right) \phi_{s}(y, w) d s\right| & \leqslant \frac{1}{N} \sum_{j=1}^{N} \alpha\left|Z_{s}^{j}-\bar{Z}_{s}^{j}\right| \\
& +\alpha\left|Z_{s}^{i}-\bar{Z}_{s}^{i}\right|+\left|\frac{1}{N} \sum_{j=1}^{N} b_{s}\left(\bar{Z}_{s}^{i}, \bar{Z}_{s}^{j}\right)\right| .
\end{aligned}
$$

It then follows from the above inequalities that

$$
\begin{aligned}
\left|X_{t}^{i}-\bar{X}_{t}^{i}\right|+\left|V_{t}^{i}-\bar{V}_{t}^{i}\right| \leqslant & \int_{0}^{t}\left|V_{s}^{i}-\bar{V}_{s}^{i}\right|+\alpha\left|Z_{s}^{i}-\bar{Z}_{s}^{i}\right| \\
& +\frac{1}{N} \sum_{j=1}^{N}\left|Z_{s}^{j}-\bar{Z}_{s}^{j}\right|+\left|\frac{1}{N} \sum_{j=1}^{N} b_{s}\left(\bar{Z}_{s}^{i}, \bar{Z}_{s}^{j}\right)\right| d s .
\end{aligned}
$$

Since the right-hand side is non-decreasing in $t$, the same estimate also holds for

$$
\sup _{0 \leqslant s \leqslant t}\left[\left|X_{s}^{i}-\bar{X}_{s}^{i}\right|+\left|V_{s}^{i}-\bar{V}_{s}^{i}\right|\right]
$$

in place of $\left|X_{t}^{i}-\bar{X}_{t}^{i}\right|+\left|V_{t}^{i}-\bar{V}_{t}^{i}\right|$. 
Recall that by symmetry of the laws of $\left(X_{t}^{i}, V_{t}^{i}\right)$ and $\left(\bar{X}_{t}^{i}, \bar{V}_{t}^{i}\right)$, one has

$$
\begin{aligned}
\mathbb{E}\left[\frac{1}{N} \sum_{i=1}^{N}\left|X_{t}^{i}-\bar{X}_{t}^{i}\right|+\left|V_{t}^{i}-\bar{V}_{t}^{i}\right|\right] & =\frac{1}{N} \sum_{i=1}^{N} \mathbb{E}\left[\left|X_{t}^{i}-\bar{X}_{t}^{i}\right|+\left|V_{t}^{i}-\bar{V}_{t}^{i}\right|\right] \\
& =\mathbb{E}\left[\left|X_{t}^{1}-\bar{X}_{t}^{1}\right|+\left|V_{t}^{1}-\bar{V}_{t}^{1}\right|\right] .
\end{aligned}
$$

Therefore, summing equalities in (6.9) over $i=1, \ldots, N$, combining all these estimates, yields

$\mathbb{E}\left[\frac{1}{N} \sum_{i=1}^{N}\left|X_{t}^{i}-\bar{X}_{t}^{i}\right|+\left|V_{t}^{i}-\bar{V}_{t}^{i}\right|\right] \leqslant 2 \max (1, \alpha) \int_{0}^{t} \mathbb{E}\left(\left|X_{t}^{i}-\bar{X}_{t}^{i}\right|+\left|V_{t}^{i}-\bar{V}_{t}^{i}\right|\right) d s+B(s) d s$,

where

$$
B(s)=\mathbb{E}\left[\frac{1}{N} \sum_{j=1}^{N} b_{s}\left(\bar{Z}_{s}^{i}, \bar{Z}_{s}^{j}\right)\right]
$$

Using Hölder's inequality we get:

$$
B^{2}(s)=\mathbb{E}\left[\left(\frac{1}{N} \sum_{j=1}^{N} b_{s}\left(\bar{Z}_{s}^{i}, \bar{Z}_{s}^{j}\right)\right)^{2}\right]=\mathbb{E}\left[\frac{1}{N^{2}} \sum_{j, k} b_{s}\left(\bar{Z}_{s}^{1}, \bar{Z}_{s}^{j}\right) b_{s}\left(\bar{Z}_{s}^{1}, \bar{Z}_{s}^{k}\right)\right] .
$$

Furthermore, direct calculation shows that

$$
\begin{aligned}
& b_{s}\left(\bar{Z}_{s}^{1}, \bar{Z}_{s}^{j}\right) b_{s}\left(\bar{X}_{s}^{1}, \bar{Z}_{s}^{k}\right)=\left(K\left(\bar{Z}_{s}^{1}, \bar{Z}_{s}^{j}\right)-\int K\left(\bar{Z}_{s}^{1}, y\right) u_{s}(y, w)\right) \\
& \times\left(K\left(\bar{Z}_{s}^{1}, \bar{Z}_{s}^{k}\right)-\int K\left(\bar{Z}_{s}^{1}-y\right) u_{s}(y, w)\right. \\
&= K\left(\bar{Z}_{s}^{1}, \bar{Z}_{s}^{j}\right) K\left(\bar{Z}_{s}^{1}-\bar{Z}_{s}^{k}\right)-K\left(\bar{Z}_{s}^{1}, \bar{Z}_{s}^{j}\right) \int K\left(\bar{Z}_{s}^{1}, y\right) u_{s}(y, w) \\
&-K\left(\bar{Z}_{s}^{1}-\bar{Z}_{s}^{k}\right) \int K\left(\bar{Z}_{s}^{1}, y\right) u_{s}(y, w)+\left(\int K\left(\bar{Z}_{s}^{1}, y\right) u_{s}(y, w)\right)^{2} .
\end{aligned}
$$

Taking the expectation of each term on the right side of the equality (6.12) and noting that, for $1 \leqslant i \leqslant N$, law of

$$
\left(\bar{X}_{t}^{i}, \bar{V}_{t}^{i}\right)=u_{i}^{\otimes N}
$$

the immediate consequences are

$$
\begin{aligned}
& \mathbb{E}\left[K\left(\bar{Z}_{s}^{1}, \bar{Z}_{s}^{j}\right) K\left(\bar{Z}_{s}^{1}, \bar{Z}_{s}^{k}\right)\right]=\int\left(K\left(z, z^{\prime}\right) K\left(z, z_{1}^{\prime}\right) \phi_{s}\left(z^{\prime}\right) \phi_{s}\left(z_{1}\right) d z=\int\left(K\left(z, z^{\prime}\right) \phi_{s}(z)^{2} \phi_{s}\left(z^{\prime}\right) d z\right.\right. \\
& \mathbb{E}\left[K\left(\bar{Z}_{s}^{1}, \bar{Z}_{s}^{j}\right) \int K\left(\bar{Z}_{s}^{1}, y\right) u_{s}(y, w)\right]=\int\left(K\left(z, z^{\prime}\right) \phi_{s}(z)^{2} \phi_{s}\left(z^{\prime}\right) d z\right. \\
& \mathbb{E}\left[K\left(\bar{Z}_{s}^{1}-\bar{Z}_{s}^{k}\right) \int K\left(\bar{Z}_{s}^{1}, y\right) u_{s}(y, w)\right]=\int\left(K\left(z, z^{\prime}\right) \phi_{s}(z)^{2} \phi_{s}\left(z^{\prime}\right) d z\right. \\
& \mathbb{E}\left[\left(\int F\left(\bar{Z}_{s}^{1}, y\right) u_{s}(y, w)\right)^{2}\right]=\int\left(K\left(z, z^{\prime}\right) \phi_{s}(z)^{2} \phi_{s}\left(z^{\prime}\right) d z .\right.
\end{aligned}
$$


Notice that when $j \neq k$, on has

$$
\mathbb{E}\left[b_{s}\left(\bar{Z}_{s}^{1}, \bar{Z}_{s}^{j}\right) b_{s}\left(\bar{Z}_{s}^{1}, \bar{Z}_{s}^{k}\right)\right]=0 .
$$

Upon placing th above informations into the right-hand side of (6.11), we obtain

$$
B^{2}(s) \leqslant \frac{1}{N^{2}} \sum_{j=1}^{N} \mathbb{E}\left(b_{s}\left(\bar{Z}_{s}^{i}, \bar{Z}_{s}^{j}\right)^{2}\right) \leqslant \frac{2}{N}\|K\|_{\infty}^{2} .
$$

When this estimate is combined with estimate (6.10) we see that

$$
\begin{aligned}
\Phi(t) & =\mathbb{E}\left[\sup _{0 \leqslant s \leqslant t} \| X_{s}^{i}-\bar{X}_{s}^{i}|+| V_{s}^{i}-\bar{V}_{s}^{i} \mid\right] \\
& \leqslant \Lambda \int_{0}^{t} \Phi(s) d s+\frac{2}{N}\|K\|_{\infty}^{2},
\end{aligned}
$$

where $\Lambda=2 \max (1, \alpha)$. Gronwall's lemma now implies

$$
\Phi(t) \leqslant \frac{2\|K\|_{\infty}}{\sqrt{N}} e^{\Lambda t},
$$

which yields the upper bound of (6.7) thereby finish the proof of Theorem 6.1.

We next interpret Theorem 6.1 as chaotic property of the diffusion governed by (6.4). The problem of chaos propagation arises when one first tried to understand the relationship between the macroscopic and microscopic evolution of a system of interacting particles. At the microscopic level, the system is modeled by a Markov process while at the macroscopic level, one uses PDE usually nonlinear. The chaos propagation property makes the connection between these two points of view. Recall that by Proposition 2.2, [30], a sequences $\left(u_{N}\right)$ of symmetric probability measures on $E^{(N)}$ is $u$-chaotic, $u \in \mathscr{M}(E)$, if for $\left(X_{1}, \ldots, X_{N}\right)$ distributed as $u_{N}$, it holds

$$
\frac{1}{N} \sum_{i=1}^{N} \delta_{X^{i}, V^{i}} \stackrel{\text { w }}{\longrightarrow} u
$$

For $\left(\bar{X}^{N}, \bar{V}^{N}\right):=\frac{1}{N} \sum_{i=1}^{N} \delta_{X^{i, N}, V^{i, N}}$, we obtain from Theorem 6.1

$$
\left(\bar{X}^{N}, \bar{V}^{N}\right) \stackrel{\text { w }}{\longrightarrow}(\bar{X}, \bar{V})
$$

where $(\bar{X}, \bar{V})$ is the solution of equation (6.5). Therefore, denoting by $m$ the law of $\bar{X}$ and by $m_{N}$ the law of $\left(X^{1, N}, \ldots, X^{N, N}\right)$, we obtain from (6.14), that under the assumptions of Theorem $6.1,\left(m_{N}\right)$ is $m$-chaotic.

In fact the bound (6.7) gives estimates on the propagation of chaos for the particles system, the convergence of the law of a single particle, and also the convergence of the empirical measure, since

$$
\mathbb{E}\left|\int_{\mathbb{R}^{2 d}} \varphi \mu_{Z_{0}^{N}}-\int_{\mathbb{R}^{2 d}} \varphi f_{t}\right| \leqslant \frac{C}{\sqrt{N}}
$$

for all 1-Lipschitz observables $\varphi$ on $\mathbb{R}^{2 d}$. 


\section{Applications}

From the modeling viewpoint, the system (1.1) is able to describe self-organized dynamics based on alignment for populations of birds or fish, pedestrians, networks, whose individuals are moving in $\mathbb{R}^{2 d}$. In that case the term $\mathbb{G}(z)$ contains the desired velocity $v_{\text {des }}$ that, without losing generality, we can assume to have constant modulus.

In particular $\mathbb{G}$ can be chosen as follows:

(1) $\mathbb{G}(z) \equiv-\nabla U(x)$ (potential force $U(x)$, i.e. $U(x)=\alpha\left|x-x_{0}\right|^{-1}$ );

(2) $\mathbb{G}(z) \equiv \alpha v$ (self-propulsion force proportional to the velocity: if it were not balanced by other terms, it would make the individuals accelerate along the velocity they have at time $t$ )

(3) $\mathbb{G}(z) \equiv \alpha\left(\beta-|v|^{2}\right) v, \alpha>0$ (self-propulsion force and friction or Rayleigh dissipation or Rayleigh-Helmholtz friction [28]).

and $K$ as follows:

(1) $K$ depends on the relative position $x_{i}-x_{j}$ only, and it derives from the potential in the form $K(x, v)=-\nabla U(x), \quad U(x)=\alpha|x|^{-1}$

(2) $K\left(z_{i}, z_{j}\right) \equiv K\left(x_{i}-x_{j}, v_{i}-v_{j}\right)=a\left(\left|x_{i}-x_{j}\right|\right)\left|v_{j}-v_{i}\right|^{p-1}$ (flocking models like Cuker-Smale [11]). Each particle attempts to align its velocity with a local average velocity given by $K$.

(3) In crowd motion $K\left(z_{i}, z_{j}\right)$ describes how the $i$ th pedestrian corrects its desired velocity because binary interactions with the $j$ th pedestrian belonging to its interaction neighborhood.

It is worth stressing that one of the possible configurations in the interaction $K$ is the case where the visual a perception cone of the single individual plays a fundamental role: individual $j$ is perceived by individual $i$ but not vice versa. Some of these models exist in the simulation of flocking and swarming dynamics. We refer the interested reader to the review papers $[1,2,6,10]$ for a general account and comparison of the existing models.

\section{Acknowledgments}

Carlo Bianca was partially supported by L'Agence Nationale de la Recherche (ANR T-KiNeT Project).

\section{References}

[1] M. Agueh, R. Illner, and A. Richardson, Analysis and simulations of a refined flocking and swarming model of Cucker-Smale type, Kinet. Relat. Models 4 (2011) 1-16.

[2] G. Albi and L. Pareschi, Binary Interaction Algorithms for the Simulation of Flocking and Swarming Dynamics, Multiscale Model. Simul. 11 1-29.

[3] L. Ambrosio, N. Gigli, and G. Savaré, Gradient Flows in Metric Space and in the Wasserstein Space of Probability Measures (Lectures in Mathematics ETH Zurich, Birkhaüser Verlag, Basel, 2008).

[4] A. J. Bernoff and Chad M. Topaz, A Primer of Swarm Equilibria, SIAM J. Appl. Dyn. Syst. 10 (2011) 212-250.

[5] M. Bodnar and J. J. Velasquez, Derivation of macroscopic equations for individual cell-based models: A formal approach, Math. Meth. Appl. Sci. 28 (2005) 1757-1779.

[6] F. Bolley, J. A. Canizo, J. A. Carrillo, Stochastic mean-field limit: non-Lipschitz forces and swarming, Math. Models and Meth. App. 21 (2011) 2179-2210.

[7] W. Braun and K. Hepp, The Vlasov dynamics and its fluctuations in the $1 / \mathrm{N}$ limit of interacting classical particles, Commun. Math. Phys. 56 (1977) 101-113. 
[8] D. R. Brillinger, A particle migrating randomly on a sphere, J. Th. Probability 10 (1997) 429-443.

[9] E. Caglioti, P. L. Lions, C. Marchioro, and M. Pulvirenti, A Special Class of Stationary Flows for TwoDimensional Euler Equations: A Statistical Mechanics Description, Commun. Math. Phys. 143 (1992) 501-525.

[10] J. A. Canizo, J. A. Carrillo, J. J. Rosado, A well-posedness theory in measures for some kinetic models of collective motion, Math. Models Methods Appl. Sci. 21 (2011) 515-539.

[11] F. Cucker and S. Smale, Emergent behavior in flocks, IEEE Trans. Automat. Control 52 (2007) 852-862.

[12] R. L. Dobrušin, Vlasov equations, Funktsional. Anal. i Prilozhen. 13 (1979) 48-58.

[13] M. D’Orsogna, Y. L. Chuang, A. Bertozzi, L. Chayes, Self-propelled particles with soft-core interactions: patterns, stability, and collapse, Physical Review Letters 96 (2006) 104302-104314.

[14] R. M. Dudley, Real Analysis and Probability (Wadsworth and Brooks/Cole, Pacific Grove, 1989).

[15] L. C. Evans, R. F. Gariepy, Measure Theory and Fine Properties of Functions, Studies in Advanced Mathematics (CRC Press, Boca Raton, FL, 1992).

[16] J. Evers, A. Muntean, Modeling micro-macro pedestrian counterflow in heterogeneous domains, Nonlinear Phenomena in Complex Systems 14 (2011) 27-37.

[17] F. Golse, The Mean-Field Limit for the Dynamics of Large Particle Systems Journées "Équations aux Dérivées Partielles (Exp. No. IX, 47 pp., Univ. Nantes, Nantes, 2003).

[18] F. Golse, On the Dynamics of Large Particle Systems in the Mean Field Limit (Lecture notes for a course at the NDNS ${ }^{+}$, Applied Dynamical Systems Summer School on "Macroscopic and large scale phenomena", 2012, Universiteit Twente, Enschede (The Netherlands)).

[19] B. D. Greenshields, A Study in Highway Capacity, Highway Research Board 14 (1935) 458.

[20] F. A. Grünbaum, Propagation of chaos for the Boltzmann equation, Arch. Rational Mech. Anal. 42 (1971) 323-345.

[21] J. Haskovec, Flocking dynamics and mean-field limit in the Cucker-Smale-type model with topological interactions, Physica D: Nonlinear Phenomena 261 (2013) 42-51.

[22] C. Lancellotti, On the Fluctuations about the Vlasov Limit for N-particle Systems with Mean-Field Interactions, J Stat Phys 136 (2009) 643-665.

[23] P.-L. Lions, Mean field games (Chap. "Mesures et distances". Course at the Collège de France, 20072008).

[24] C. Mouhot, S. Michler, B. Wennber, A new approach to quantitative propagation of chaos for drift, diffusion and jump processes. Preprint, to appear in Probability Theory and Related Fields.

[25] H. Neunzert, Fluid Dyn. Trans. 9 (1977), 229.

[26] H. Niwa, Self-organizing dynamic model of fish schooling, J. Theoret. Biol. 171 (1994) 123-136.

[27] L. Onsager, Statistical hydrodynamics, Supplemento al Nuovo Cimento 6 (1949), 279-287.

[28] J. Rayleigh, The Theory of Sound (2nd ed., MacMillan, London, 1894).

[29] A.-S. Sznitman, Equations de type de Boltzmann, spatialement homogènes, Z. Wahrsch. Verw. Gebiete 66 (1984) 559-592.

[30] A.-S. Sznitman, Topics in propagation of chaos (Lecture Notes in Math. 1464, Springer, Berlin, 1991).

[31] C. Villani, Optimal Transport, Old and new. Fundamental Principles of Mathematical Sciences (Springer-Verlag, Berlin, 2009). 